\title{
Electronic and magnetic properties of $\mathrm{K}_{2} \mathrm{CuP}_{2} \mathrm{O}_{7}$ - a model $\mathrm{S}=1 / 2$ Heisenberg chain system
}

\author{
R. Nath, Deepa Kasinathan, H. Rosner * M. Baenitz, and C. Geibel \\ Max Planck Institut für Chemische Physik fester Stoffe, Nöthnitzer Str. 40, 01187 Dresden, Germany
}

(Dated: November 11, 2018)

\begin{abstract}
The electronic and magnetic properties of $\mathrm{K}_{2} \mathrm{CuP}_{2} \mathrm{O}_{7}$ were investigated by means of susceptibility, specific heat and ${ }^{31} \mathrm{P}$ nuclear magnetic resonance (NMR) measurements and by LDA band structure calculations. The temperature dependence of the NMR shift $K(T)$ is well described by the $S=\frac{1}{2}$ Heisenberg antiferromagnetic chain (HAF) model with nearest neighbor exchange $J_{1} \simeq(141 \pm 5)$ $\mathrm{K}$. The corresponding mapping of an LDA-derived tight-binding model leads to $J_{1}^{L D A} \simeq 196 \mathrm{~K}$. The spin lattice relaxation rate $1 / T_{1}$ decreases with temperature below $300 \mathrm{~K}$ but becomes nearly temperature independent between $30 \mathrm{~K}$ and $2 \mathrm{~K}$ as theoretically expected for an $S=\frac{1}{2}$ HAF chain. None of the investigated properties give any evidence for long range magnetic order above $2 \mathrm{~K}$, in agreement with the results of the band structure calculation, which yield extremely weak exchange to the next nearest neighbor (NNN) and a very small and frustrated inter-chain exchange. Thus, $\mathrm{K}_{2} \mathrm{CuP}_{2} \mathrm{O}_{7}$ seems to be a better realization of a nearest neighbor $S=\frac{1}{2} \mathrm{HAF}$ chain than the compounds reported so far.
\end{abstract}

PACS numbers: 75.10.Pq, 75.40.Cx, 76.60.-k, 76.60.Cq

\section{INTRODUCTION}

One-dimensional (1D) spin systems have attracted considerable attention because of their intriguing ground states where quantum fluctuations play a crucial role. Much excitement in their magnetism has been caused by the theoretical prediction that the integer-spin chains have an energy gap in the excitation spectrum,,$\frac{1}{\text { - while }}$ the half-integer spin chains have a gapless excitation spectrum ${ }^{2.3}$ In particular, $1 \mathrm{D} S=\frac{1}{2}$ Heisenberg antiferromagnetic (HAF) systems are interesting since enhanced quantum fluctuations due to reduced dimensionality and low spin value impede long range magnetic order (LRO). In the last decade, theoretical studies on these systems have achieved a remarkable progress but real material realizations for such compounds are limited to date.

The $S=\frac{1}{2}$ chains formed via direct linkage of $\mathrm{CuO}_{4}$ units can be grouped into two categories: in one group, the chains are formed by edge-sharing $\mathrm{CuO}_{4}$ units while in the other compound family they are built from cornersharing $\mathrm{CuO}_{4}$ units. $\mathrm{CuGeO}_{3}$ and $\mathrm{Li}_{2} \mathrm{CuO}_{2}$ belong to the former category ${ }^{4,5}$, , where the nearest neighbor $(\mathrm{NN})$ interaction $J_{1}$ and the next-nearest neighbor (NNN) interaction $J_{2}$ have comparable strength $\left(\frac{\left|J_{2}\right|}{\left|J_{1}\right|} \approx 1\right)$ thereby leading to a strong frustration within the chain. In such a scenario, various ground states are possible. On the contrary, in $\mathrm{Sr}_{2} \mathrm{CuO}_{3}$ which has corner shared $\mathrm{CuO}_{4}$ units $\frac{\left|J_{2}\right|}{\left|J_{1}\right|} \approx \frac{1}{20}$, which greatly reduces the in-chain frustration 6.7 .8 . With $J_{1} \gg \mathrm{T}_{N}, \mathrm{Sr}_{2} \mathrm{CuO}_{3}$ is a better quasi-1D $S=\frac{1}{2}$ chain system than $\mathrm{CuGeO}_{3}$ and $\mathrm{Li}_{2} \mathrm{CuO}_{2}$. Recently another system, $\mathrm{Sr}_{2} \mathrm{Cu}\left(\mathrm{PO}_{4}\right)_{2}$ containing isolated $\mathrm{CuO}_{4}$ units (neither edge- nor cornershared) was reported to have $\frac{J_{2}}{J_{1}} \approx \frac{1}{700}$ and a much reduced $T_{N}$ of $85 \mathrm{mK}^{9,10,11,12}$. The presence of interchain coupling (ic) normally provides LRO, while frustrating ic reduces the tendency to LRO. Along with $J_{1}$ $\gg T_{N}$, the presence of frustration between the chains strongly influences the 1D nature of this system, making it a nearly perfect realization for the $1 \mathrm{D}$ NN only HAF chain model. Unfortunately the sample quality was not very good, thereby reducing the chains to a finite length. The analytical solution by Bethe ansatz ${ }^{2}$ provides a clear picture of the magnetic (thermodynamic) properties of a very good 1D material. Thus, deviations from these predictions can be assigned to other degrees of freedom (anisotropy, Dzyaloshinskii-Moriya (DM) and other spinspin interactions) allowing to analyse their influence on the ground state behaviour. Therefore, strong interest still prevails in the experimental community to find possible ways to synthesize a system which keeps the in-chain geometry of $\mathrm{Sr}_{2} \mathrm{Cu}\left(\mathrm{PO}_{4}\right)_{2}$, but with a smaller inter-chain coupling which is still frustrating, along with an improved sample quality.

Formaly, $\mathrm{K}_{2} \mathrm{CuP}_{2} \mathrm{O}_{7}$ belongs to the family of $\mathrm{M}_{2} \mathrm{CuP}_{2} \mathrm{O}_{7}(\mathrm{M}=\mathrm{Li}, \mathrm{Na}, \mathrm{K})$ compounds, though the arrangement of the magnetic $\mathrm{CuO}_{4}$ building blocks is modified within the family. The magnetic properties of $\mathrm{Na}_{2} \mathrm{CuP}_{2} \mathrm{O}_{7}$ and $\mathrm{Li}_{2} \mathrm{CuP}_{2} \mathrm{O}_{7}$ were reported earlier ${ }^{15.16}$ $\mathrm{Na}_{2} \mathrm{CuP}_{2} \mathrm{O}_{7}$ and $\mathrm{Li}_{2} \mathrm{CuP}_{2} \mathrm{O}_{7}$ both have an in-chain exchange coupling $J_{1}$ of about $28 \mathrm{~K}$ and they undergo magnetic ordering at around $5 \mathrm{~K}$. Unlike the other members of this family, our experiments reveal that $\mathrm{K}_{2} \mathrm{CuP}_{2} \mathrm{O}_{7}$ shows a much stronger in-chain exchange interaction $\left(J_{1} \simeq 141 \mathrm{~K}\right)$ but no ordering down to $2 \mathrm{~K}$. Due to such a wide $T_{N^{-}} J_{1}$ temperature range it can be considered as a very good candidate on which to test the theoretical predictions of both static and dynamic properties of an 1D HAF model.

In this paper, we present susceptibility, specific heat, ${ }^{31} \mathrm{P}$ NMR results and first principles band structure calculations on $\mathrm{K}_{2} \mathrm{CuP}_{2} \mathrm{O}_{7}$ in order to shed light on the $1 \mathrm{D}$ character of the system. The manuscript is organized as follows: In the next two sections, we describe the struc- 


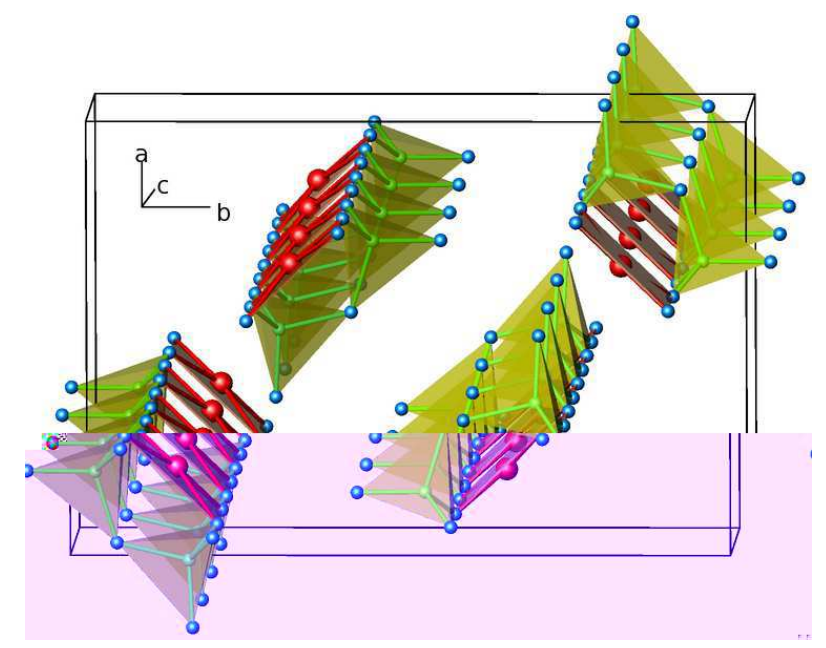

FIG. 1: $\mathrm{K}_{2} \mathrm{CuP}_{2} \mathrm{O}_{7}$ crystal structure. The $\mathrm{CuO}_{4}$ square planes (red) share their edges with the $\mathrm{PO}_{4}$ tetrahedra (green) to form $\left[\mathrm{Cu}\left(\mathrm{PO}_{4}\right)_{2}\right]_{\infty}$ linear chains propagating along $c$ direction. The potassium cations reside in between the chains (not shown here).

tural aspects, measurement procedures and the theoretical methodology. In section IV we present the experimental results followed by the electronic structure analysis. In the discussion section, we address the issues concerning the almost ideal 1D nature of this compound inferred from the ${ }^{31} \mathrm{P}$ NMR and band structure calculations. At the end we give a short summary and conclusions.

\section{STRUCTURE}

$\mathrm{K}_{2} \mathrm{CuP}_{2} \mathrm{O}_{7}$ crystallizes in an orthorhombic unit cell with space group Pbnm. The reported lattice constants are $a=9.509 \AA, b=14.389 \AA$, and $c=5.276 \AA .14$ In the crystal structure, isolated quasi planar $\mathrm{CuO}_{4}$ units are linked by $\mathrm{PO}_{4}$ tetrahedra, forming $\left[\mathrm{Cu}\left(\mathrm{PO}_{4}\right)_{2}\right]_{\infty}$ chains propagating along the crystallographic $c$-direction. A scheme of the basic building blocks of the crystal is shown in Fig. 1. The super-exchange between $\mathrm{Cu}^{2+}$ ions in $\mathrm{K}_{2} \mathrm{CuP}_{2} \mathrm{O}_{7}$ will be similar to that of edge sharing $\mathrm{CuO}_{4}$ chains with every second $\mathrm{CuO}_{4}$ unit cut-off. In such a situation, $J_{2}(\mathrm{NNN})$ in the edge shared system will become $J_{1}(\mathrm{NN})$ in our system (of the order of $\approx 100 \mathrm{~K}$ ), and $J_{2}$ in our system will be comparable to $J_{4}$ (fourth neighbor interaction along the chain) in the edge shared system, which is known to be quite negligible. The chains are well separated from each other since the potassium cations, $\mathrm{K}^{1+}$ reside in between the chains. The interchain interactions are expected to be very weak, unlike $\mathrm{Sr}_{2} \mathrm{Cu}\left(\mathrm{PO}_{4}\right)_{2}$ since the chains do not lie in the same plane. Magnetic properties of this compound have not been reported yet.

\section{METHODS}

\section{A. Experimental}

Polycrystalline $\mathrm{K}_{2} \mathrm{CuP}_{2} \mathrm{O}_{7}$ was prepared by solid state reaction techniques using $\mathrm{K}_{2} \mathrm{CO}_{3}$ (99.9\% pure), $\mathrm{CuO}$ (99.99\% pure) and $\mathrm{NH}_{4} \mathrm{H}_{2} \mathrm{PO}_{4}(99.9 \%$ pure) as starting materials. The stoichiometric mixtures were fired at $640^{\circ} \mathrm{C}$ for 60 hours in air, with one intermediate grinding. The samples were characterized using a STOE powder diffractometer with a $\mathrm{Cu}$ target $\left(\lambda_{a v}=1.54182 \AA\right)$. The powder pattern evidenced single phase material. The lattice parameters obtained using a least-square fit procedure are $a=9.541(3) \AA, b=14.407(5) \AA, c=5.253(1)$ $\AA$, close to the previously reported values 14

Magnetization $(M)$ data were measured as a function of temperature $T$ between $2 \mathrm{~K}$ and $400 \mathrm{~K}$ in fields up to 5 $\mathrm{T}$ on powder samples in a commercial (Quantum design) SQUID (superconducting quantum interference device) magnetometer. Specific heat $C_{p}(T)$ measurements were performed on a pressed pellet using relaxation method in a commercial PPMS equipment(Quantum design). NMR measurements were carried out using pulsed NMR techniques on ${ }^{31} \mathrm{P}$ nuclei (nuclear spin $I=\frac{1}{2}$ and gyromagnetic ratio $\gamma / 2 \pi=17.237 \mathrm{MHz} /$ Tesla) at $70 \mathrm{MHz}$ which corresponds to an applied field of about $40.6 \mathrm{kOe}$. Spectra were obtained by Fourier transform (FT) of the NMR echo signal using a $\pi / 2$ pulse with width of about $2 \mu \mathrm{s}$. The NMR shift $K(T)=\left[\nu(T)-\nu_{\text {ref }}\right] / \nu_{\text {ref }}$ was determined by measuring the resonance frequency of the sample $(\nu(T))$ with respect to a standard $\mathrm{H}_{3} \mathrm{PO}_{4}$ solution (resonance frequency $\nu_{\text {ref }}$ ). The spin-lattice relaxation rate $\left(1 / T_{1}\right)$ was measured by the saturation recovery method. Our attempt to see the ${ }^{63} \mathrm{Cu}$ signal was not successful due to the fast relaxation at the magnetic site.

\section{B. Theory}

The bandstructure calculations presented here utilized version 5.00-18 of the full-potential local orbital band structure 17.18 (FPLO) method. The structure, lattice constants and atomic positions have been taken from experiment ${ }^{14}$. The core states have been treated fullyrelativistically while the semi-core $(\mathrm{K}: 3 s 3 p, \mathrm{Cu}: 3 s 3 p$, P: $2 s 2 p$ ) and the valence states (K: $3 d 4 s 4 p, \mathrm{Cu}: 3 d 4 s 4 p$, P: $3 s 3 p 3 d$, O: $2 s 2 p 3 d$ ) are treated scalar-relativistically. The extent of the valence basis functions was optimized with respect to the total energy ${ }^{19}$. The Brillouin zone sampling was based on $216 k$-points in the irreducible wedge. We have used the Perdew and Wang ${ }^{20}$ flavor of the exchange and correlation potential when performing the calculations within the local density approximation (LDA). 


\section{RESULTS}

\section{A. Susceptibility and specific heat}

Bulk magnetic susceptibility $\chi(T)(=M / H)$ was measured as a function of temperature in an applied field of $5 \mathrm{kOe}$ (Fig. 2). As shown in the figure, the sample exhibits a shoulder at about $100 \mathrm{~K}$, suggesting a maximum in this temperature range, which is a hallmark of low-dimensional magnetic interactions. With further decreasing temperature, $\chi(T)$ increases again in a Curie-Weiss manner. Common sources for such an increase are the presence of paramagnetic impurities or finite length chains due to disorder. However, an intrinsic Curie like tail is expected in a quasi $1 \mathrm{D} S=\frac{1}{2}$ chain, if DM interaction and/or a staggered $g$-factor anisotropy are present. ${ }^{21,22}$ Among the $3 \mathrm{~d}$ systems, $\mathrm{Cu}$ benzoate ${ }^{23}$ and pyrimidine $\mathrm{Cu}$ dinitrate ${ }^{24}$ are well known examples. No obvious features associated with LRO were observed for $1.8 \mathrm{~K} \leq T \leq 400 \mathrm{~K}$.

In order to fit the bulk susceptibility data, we decomposed $\chi$ into

$$
\chi=\chi_{0}+\frac{C_{i m p}}{T+\theta_{i m p}}+\chi_{s p i n}(T)
$$

where, the first term $\chi_{0}$ is temperature independent and accounts for the diamagnetism of the core electron shells and Van-Vleck paramagnetism of the open shells of the $\mathrm{Cu}^{2+}$ ions. The second term $\frac{C_{i m p}}{T+\theta_{i m p}}$ is the low $T$ CurieWeiss contribution due to paramagnetic species in the

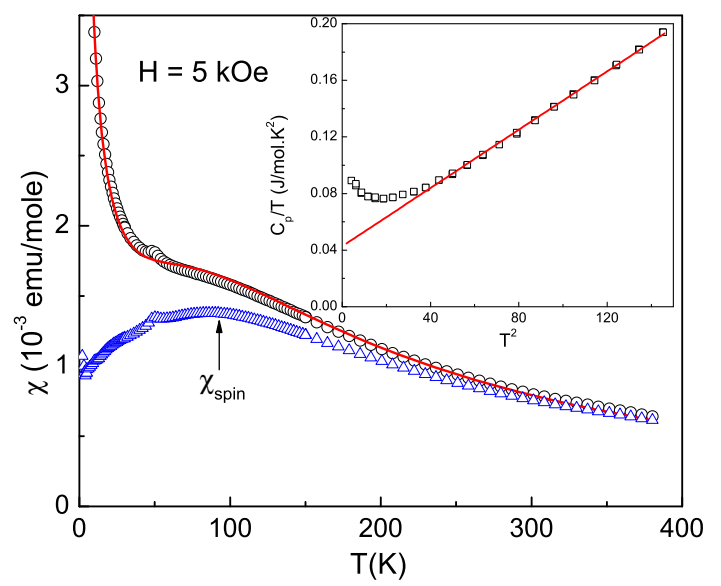

FIG. 2: Magnetic susceptibility $(M / H)$ vs. temperature $T$ for $\mathrm{K}_{2} \mathrm{CuP}_{2} \mathrm{O}_{7}$ (open circles) in an applied field of $5 \mathrm{kOe}$. The solid line is best fit of the data to Eq. 1. Spin susceptibility $\chi_{\text {spin }}$ is plotted (open triangles) after subtracting the Curie contribution. In the inset, $C_{p} / T$ is plotted as a function of $T^{2}$ for the low temperatures. Solid line represents a linear fit. sample. $\chi_{\text {spin }}(T)$ is the spin susceptibility for a uniform $S=\frac{1}{2} 1 \mathrm{D}$ HAF system, which is known quite precisely over the whole measured temperature range. We took the Johnston's expression 26 valid for $5 \times 10^{-25} \leq \frac{T}{J_{1}} \leq 5$.

A fit of Eq. 1 to the experimental data leads to the parameters $\chi_{0} \simeq 2.7 \times 10^{-4} \mathrm{emu} / \mathrm{mole}, C_{i m p} \simeq 0.026$ emu.K/mole, $\theta_{i m p} \simeq 0.7 \mathrm{~K}$, and $J_{1} \simeq 145 \mathrm{~K}$. Because of large Curie tail, such a fit is unstable at high temperatures. Therefore we reduced the number of fitting parameters by fixing $g=2.2$ (obtained from NMR shift analysis $)^{27}$. The value of $\chi_{0}$ is comparable to that found in $\mathrm{Sr}_{2} \mathrm{CuO}_{3}{ }^{7}$. The Curie contribution present in the sample would correspond to a defect spin concentration of 6.9 $\%$ assuming defect spin $S=\frac{1}{2}$. Alternatively, it would correspond to the contribution expected for finite chains with a length of $\approx 15$ spins according to the calculation of Schmidt et al., 28 . We add in Fig. 2 a plot of $\chi_{\text {spin }}$ as a function of temperature, after subtracting the Curie contribution. Now a pronounced broad maximum around $100 \mathrm{~K}$ is well resolved. The small peak at $50 \mathrm{~K}$ corresponds to a parasitic contribution of adsorbed oxygen . Unfortunately, we failed to improve the quality of the sample, since further annealing (in flowing Ar or vacuum) did not lead to any noticeable change in the XRD pattern.

The information about the lattice dimensionality can be obtained from the low temperature specific heat $C_{p}(T)$. For a $S=\frac{1}{2}$ chain, one expects a linear term, whereas for a square lattice, one expects a leading quadratic term in the absence of excitation gap. In the $C_{p} / T$ vs. $T^{2}$ plot (shown in the inset of Fig. 2) in the temperature range $5 \mathrm{~K} \leq T \leq 12 \mathrm{~K}$, one can see clearly that the plot follows a straight line over a considerable temperature range. This indicates that $C_{p}(T)$ is a sum of a linear and a cubic contribution. Since the cubic term corresponds to the expected contribution of the phonons, this demonstrate that the leading term of the magnetic contribution is linear in $T$. For an $S=\frac{1}{2}$ HAF chain, theoretical calculations ${ }^{26,29}$ predict for low temperatures $\left(T<0.2 J_{1}\right) ; \frac{C_{p}}{T}=\frac{2 R}{3 J_{1}}=\gamma_{\text {theo. }}$. With $J_{1} \simeq 141 \mathrm{~K}$ obtained from the NMR shift $K(T)$ analysis (presented later), this corresponds to a value $\gamma_{\text {theo }} \simeq 0.04$ $\mathrm{J} / \mathrm{K}^{2}$ mole. Fitting the measured data in the range $5 \mathrm{~K}$ $\leq T \leq 12 \mathrm{~K}$, we obtained $\gamma_{\exp } \simeq 0.042 \mathrm{~J} / \mathrm{K}^{2}$ mole. The value of $\gamma_{\text {exp }}$ is close to the value predicted theoretically. This strongly supports the quasi 1D nature of the spin system in $\mathrm{K}_{2} \mathrm{CuP}_{2} \mathrm{O}_{7}$. No other anomaly was observed down to $2 \mathrm{~K}$ in $C_{p}(T)$, suggesting the absence of magnetic order. Below $5 \mathrm{~K}$, our experimental data deviates upward from the straight line fit (inset Fig. 2). This is likely related to the contributions of paramagnetic impurities and of chain ends, which should follow a $C \approx A / T^{2}$ behavior. 


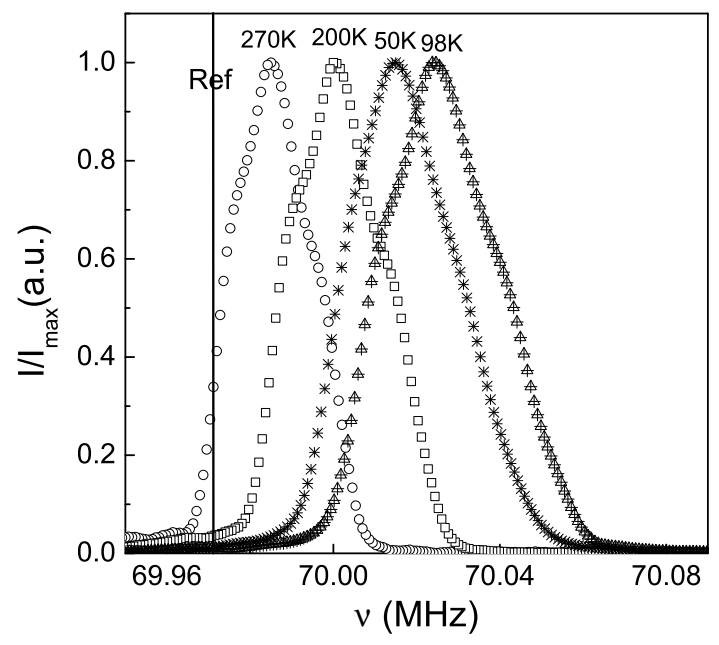

FIG. 3: ${ }^{31} \mathrm{P}$ NMR spectra at different temperatures $T$. Solid line represents the nonmagnetic ${ }^{31} \mathrm{P}$ reference.

\section{B. ${ }^{31} \mathbf{P}$ NMR}

Although our analysis of the susceptibility and specific heat suggests the presence of a $S=\frac{1}{2} 1 \mathrm{D} \mathrm{HAF}$ system, the evidence is only of preliminary nature because of the large Curie tail in $\chi(T)$ at low $T$. In order to gain a more reliable insight into this system, we turned our attention towards NMR results. NMR has the advantage to be much less sensitive to contribution of defects or impurities, because usually only the nucleus on an undistorted site contributes to the narrow NMR line. As shown in the crystal structures (Fig. 1), $\mathrm{K}_{2} \mathrm{CuP}_{2} \mathrm{O}_{7}$ has two inequivalent ${ }^{31} \mathrm{P}$ sites which are coupled inductively to $\mathrm{Cu}^{2+}$ ions in the chain. Therefore ${ }^{31} \mathrm{P}$ NMR can probe accurately the low-lying excitations in the spin chain. Our ${ }^{31} \mathrm{P}$ NMR spectra consist of a single spectral line as is expected for $I=\frac{1}{2}$ nuclei (Fig. 3). Although there are two inequivalent ${ }^{31} \mathrm{P}$ sites present the crystal structure, a single resonance line implies that both the ${ }^{31} \mathrm{P}$ sites in this compound are nearly identical. With decreasing temperature, the NMR line shifts away from the Larmor frequency, broadens paramagnetically but the overall line shape remain same down to $2 \mathrm{~K}$. The asymmetric shape of the spectra corresponds to a powder pattern due to an asymmetric hyperfine coupling.

The temperature dependence of the NMR shift $K$ is shown in Fig. 4. With decreasing temperature $K(T)$ increases paramagnetically, then passes through a broad maximum at $110 \mathrm{~K}$, which is an indicative of short-range correlations, and decreases again smoothly towards low temperatures. Below $\frac{T}{J_{1}} \simeq 0.028, K(T)$ shows a much steeper decrease towards zero. As mentioned before the NMR has an advantage over bulk susceptibility. One measures accurately the $\chi_{\text {spin }}$ by NMR shift without suf-

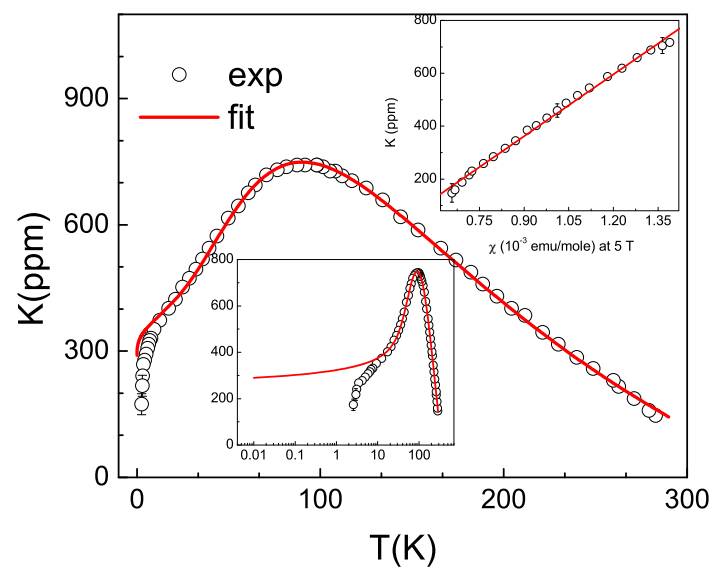

FIG. 4: Temperature dependence of the ${ }^{31} \mathrm{P}$ NMR shift $K(T)$ of $\mathrm{K}_{2} \mathrm{CuP}_{2} \mathrm{O}_{7}$. The solid line is the fit with Eq. 2 in the temperature range, $9 \mathrm{~K} \leq T \leq 300 \mathrm{~K}$ and further extrapolated down to $0 \mathrm{~K}$. Lower inset shows $K$ vs. $T$ on a logarithmic temperature scale for improved visualisation of the low- $T$ data. In the upper inset, $K$ vs. $\chi$ is plotted with temperature as an implicit parameter and the solid line is the linear fit.

fering from the contribution from the free spins and extrinsic foreign phases, which limits the accuracy of the bulk susceptibility measurements. Therefore, it is more reliable to extract the magnetic parameters from the temperature dependence of the NMR shift rather than from the bulk susceptibility. The conventional scheme of correlating $K(T)$ and $\chi(T)$ is to plot, $K$ vs. $\chi_{\text {spin }}$ with $T$ as an implicit parameter. Then the slope yields the average hyperfine coupling $A_{h f}$ between the ${ }^{31} \mathrm{P}$ nucleus and the two nearest-neighbor $\mathrm{Cu}^{2+}$ ions. In the case of $\mathrm{K}_{2} \mathrm{CuP}_{2} \mathrm{O}_{7}$, because of the low $T$ Curie-tail in $\chi(T)$ this $K(T)$ vs $\chi(T)$ plot shows a straight line only for $T>$ $110 \mathrm{~K}$ (see upper inset of Fig. 4. Nevertheless, we can estimate $A_{h f} \approx(4400 \pm 400) \mathrm{Oe} / \mu_{B}$, which is about two times stronger than in other phosphate systems ${ }^{9.13}$. We then determine $J_{1}$ and $g$ by fitting the temperature dependence of $K$ to the following equation,

$$
K=K_{0}+\left(\frac{A_{h f}}{N \mu_{B}}\right) \chi_{s p i n}\left(T, J_{1}\right)
$$

where $K_{0}$ is the temperature independent chemical shift. As shown in Fig. 4, the $K(T)$ data fit rather well to Eq. 2 in the temperature range $9 \mathrm{~K} \leq T \leq 300 \mathrm{~K}$. Using $A_{h f} \simeq 4400 \mathrm{Oe} / \mu_{B}$ (obtained from the $\bar{K}$ vs $\chi$ analysis) we obtained $K_{0} \simeq-890 \mathrm{ppm}, J_{1} \simeq(141 \pm 5) K$, and $g \simeq$ 2.2. Below about $8 \mathrm{~K}, K(T)$ show a significant deviation from the fit (see lower inset of Fig. 4) which shall be discussed later.

The temperature dependence of ${ }^{31} \mathrm{P} 1 / T_{1}$ is presented in Fig. 5. For a $I=\frac{1}{2}$ nucleus the recovery of the longitudinal magnetization is expected to follow a single 
exponential behavior. In the experiment, we indeed observed single exponential behaviour down to $30 \mathrm{~K}$ while below $30 \mathrm{~K}$ it fitted nicely to the stretch exponential with a reduced exponent. We didn't observe any anomaly or divergence in $1 / T_{1}(T)$ down to $2 \mathrm{~K}$ which indicates the absence of magnetic ordering. For $2 \mathrm{~K} \leq T \leq 30 \mathrm{~K}, 1 / T_{1}$ almost remains constant while for $T \geq 30 \mathrm{~K}$, it increases strongly with temperature. A slight shoulder is visible in the plot $1 / T_{1}$ versus $\ln T$ around $180 \mathrm{~K}$, i.e just above $J_{1}$, suggesting a regime crossover in this temperature range.

\section{First Principles and Tight Binding}

Collected in Fig. 6 are the non-magnetic band structure and the density of states (DOS). The antibonding band is made from a half-filled $\mathrm{Cu}-3 d_{x^{2}-y^{2}}-\mathrm{O}$ $2 p_{\sigma}$ molecular orbital belonging to the $\mathrm{CuO}_{4}$ plaquettes. The band structure shows strong dispersion of about 55 $\mathrm{meV}$ parallel to the chain direction $\Gamma Z, X A$, but is nearly dispersion-less within the crystallograhic $a-b$ plane (i.e. perpendicular to the direction of the chains), indicative of the strong $1 \mathrm{D}$ character in this system. Within LDA, we get a metallic behavior, though the system is an insulator, suggested by its blue color $\stackrel{14}{*}$ This is a well known artifact of LDA wherein the effect of strong correlations is under estimated. Including the strong correlations for the $\mathrm{Cu} 3 d$ states will open up the insulating gap. This can be achieved via two possible ways: (a) performing an LDA $+U$ calculation self-consistently, (b) mapping the results from LDA first to a tight-binding model (TBM). The TBM is then mapped on to a Hubbard model, and subsequently to a Heisenberg model because the system belongs to the strong correlation limit $U \gg \mathrm{t}(\mathrm{t}$ is the leading transfer integral) at half filling.

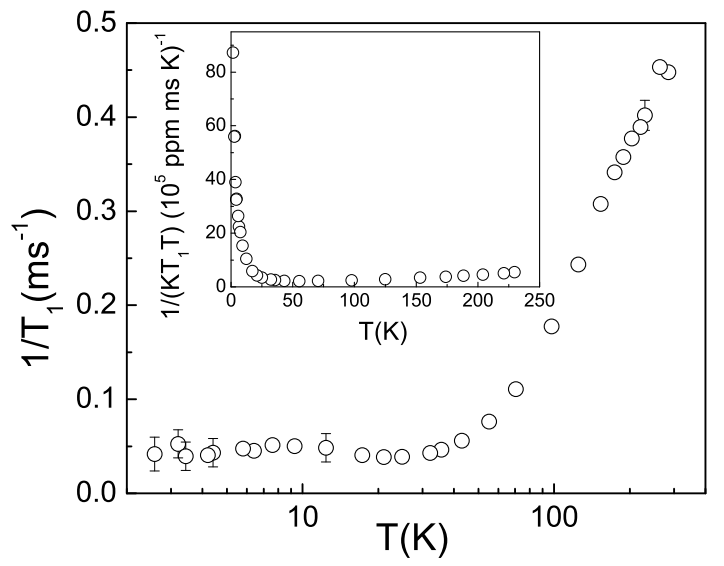

FIG. 5: Spin-lattice relaxation rate $1 / T_{1}$ vs. temperature $T$ measured at $70 \mathrm{MHz}$. In the inset, $1 /\left(K T_{1} T\right)$ is shown all over the temperature range.

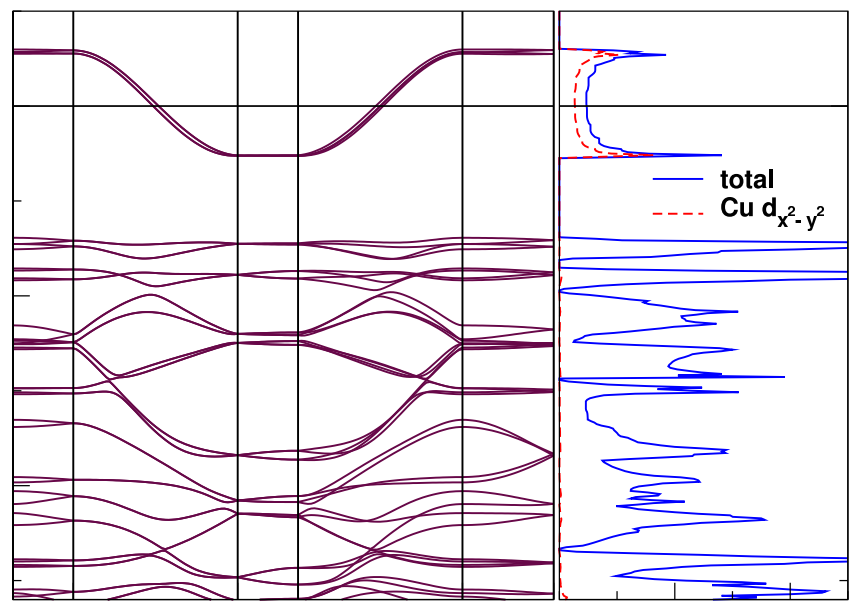

FIG. 6: (color online) Left panel: The non-magnetic band structure of $\mathrm{K}_{2} \mathrm{CuP}_{2} \mathrm{O}_{7}$ along the high-symmetry directions of a standard orthorhombic unit cell. $X-A$ and $Z-\Gamma$ denote the directions along the chain, (i.e. along crystallographic $c$ axis) wherein the dispersion is the largest while the perpendicularto-chain directions $\Gamma-X$ and $\Gamma-Y$ are rather non-dispersive. There are four copper sites per unit cell leading to four halffilled metallic bands at the Fermi level, well separated from all other bands. Right panel: The total DOS for $\mathrm{K}_{2} \mathrm{CuP}_{2} \mathrm{O}_{7}$ along with the $C u-d_{x^{2}-y^{2}}$ orbitally resolved contributions. Comparison with the band structure clearly elucidates that the half-filled metallic band at the Fermi level is primarily from $C u-d_{x^{2}-y^{2}}$ and $O-p_{\sigma}$ molecular plaquette orbital.

In order to better understand the microscopic magnetic interactions in this system, we have followed the second option mentioned above. Firstly we have considered the TBM,

$$
H=\sum_{\langle i, j\rangle, \sigma} t_{i j} C_{i, \sigma}^{\dagger} C_{j, \sigma}+\sum_{i} \epsilon_{i} \hat{n}_{i}
$$

where $t_{i j}$ are the hopping integrals, $C_{i, \sigma}^{\dagger} C_{j, \sigma}$ are annihilation and creation operators. From the LDA band structure, we have extracted only the 4 antibonding $\mathrm{Cu}$ - $3 d_{x^{2}-y^{2}}$ bands and performed a fit to the TBM. All the hopping paths considered in our model are shown in Fig. 7 The hopping integrals were calculated using the steepest descent method. The resulting parameters which provided the best fit to the LDA band structure (Fig. 8) are collected in Table [1. The strength of the NN hopping $t_{1}$ along the chain is two orders of magnitude larger than all the other hoppings, attributing the strong one-dimensionality to the interaction of the plaquettes along the chain. The individual exchange constants are calculated using $J_{i j}^{A F M}=4 t_{i j}^{2} / U_{\text {eff }}$. The value of $U_{\text {eff }}$ for $\mathrm{K}_{2} \mathrm{CuP}_{2} \mathrm{O}_{7}$ is set to $4.5 \mathrm{eV}$, same as the choice in related $1 \mathrm{D}$ compounds $(\mathrm{Sr}, \mathrm{Ba})_{2} \mathrm{Cu}\left(\mathrm{PO}_{4}\right)_{2}{ }_{21}$. The total exchange constant is given by $J^{\text {total }}=J^{A F M}+J^{F M}$.

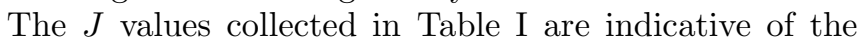
$J^{A F M}$ only. In $\mathrm{K}_{2} \mathrm{CuP}_{2} \mathrm{O}_{7}$ the $\mathrm{CuO}_{4}$ plaquettes are sep- 
arated from each other, with no corner- or edge-sharing oxygens. The absence of direct connections between the plaquettes largely suppresses ferromagnetic interactions between the copper sites. The strength of the FM interactions was shown to be very small in the related systems $(\mathrm{Sr}, \mathrm{Ba})_{2} \mathrm{Cu}\left(\mathrm{PO}_{4}\right)_{2} \frac{11}{2}$, and this result should equally hold for our system.

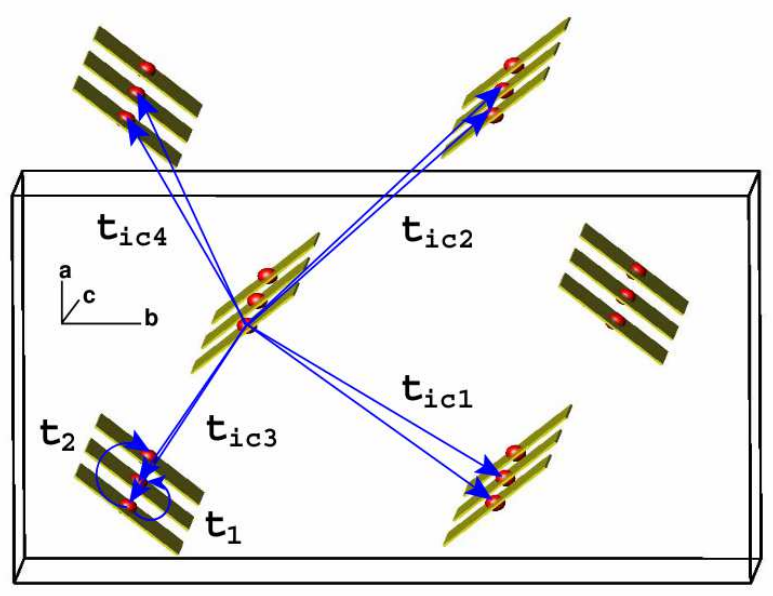

FIG. 7: (color online) The various hopping paths considered in our tight-binding model to reproduce the half-filled metallic LDA band are shown here. We have considered two hoppings along the chain $\left(t_{1}, t_{2}\right)$ and four inter-chain hoppings $\left(t_{i c 1}, t_{i c 2}, t_{i c 3}, t_{i c 4}\right)$ in all. Starting from one chain, $\mathrm{Cu}$ in the next neighbor chain are shifted by half of the in-chain $\mathrm{Cu}-\mathrm{Cu}$ distance, leading to two identical interactions to two $\mathrm{Cu}$-spins in each adjacent chains.

\begin{tabular}{|c|r|r|r|r|r|r|}
\hline & $t_{1}$ & $t_{2}$ & $t_{i c 1}$ & $t_{i c 2}$ & $t_{i c 3}$ & $t_{i c 4}$ \\
\hline$(\mathrm{meV})$ & 138 & 2 & 2 & 2 & 0.8 & 4 \\
\hline
\end{tabular}

\begin{tabular}{|l|c|c|c|c|c|c|}
\hline & $J_{1}^{L D A}$ & $J_{2}^{L D A}$ & $J_{i c 1}^{L D A}$ & $J_{i c 2}^{L D A}$ & $J_{i c 3}^{L D A}$ & $J_{i c 4}^{L D A}$ \\
\hline$(\mathrm{K})$ & 196 & 0.04 & 0.04 & 0.04 & 0.007 & 0.16 \\
\hline
\end{tabular}

TABLE I: Hopping parameters (in meV) and the corresponding exchange constants $J$ ( in $\mathrm{K}$ ) from an effective one-band tight-binding model. The hopping paths used are indicated in Fig. 7

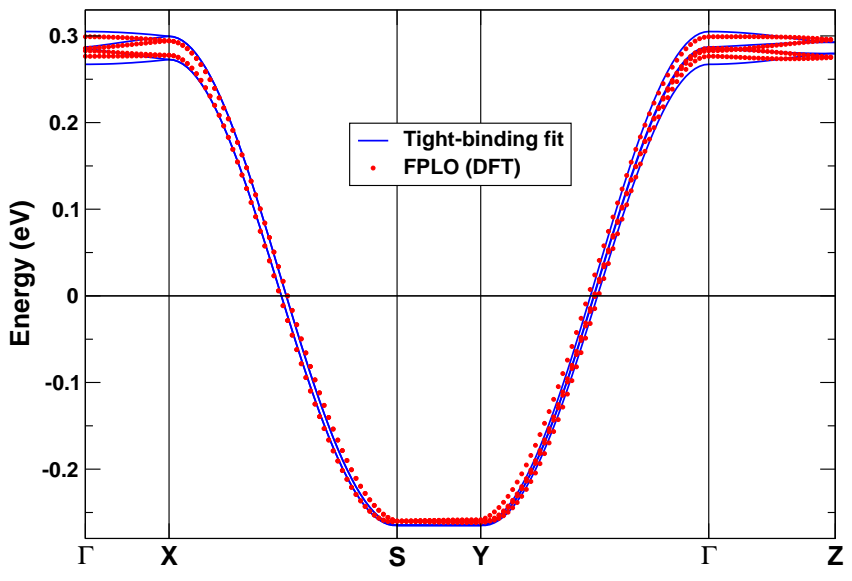

FIG. 8: (color online) The superposition of the total band structure from the FPLO density functional theory (DFT) calculations along with the calculated eigenvalues from the four-site one-band TBM. The TBM fit is quite consistent with the DFT results.

\section{DISCUSSION}

The quality of the fit for the NMR shift supports the presence of an $S=\frac{1}{2} \mathrm{HAF}$ chain. The exchange coupling $\left(J_{1} \simeq 141 \mathrm{~K}\right)$ is comparable to that found in other phosphates $\frac{9}{\underline{\underline{a}}}$ and in nice agreement with the effective NN super-exchange constant, $J_{1}^{L D A}=196 \mathrm{~K}$ obtained from the TBM. The slight over-estimation may stem from the fact that $U_{\text {eff }}$ is not exactly known, along with some FM contributions. Such a over-estimation of $J$ by LDA is well known and is also observed in $(\mathrm{Sr}, \mathrm{Ba})_{2} \mathrm{Cu}\left(\mathrm{PO}_{4}\right)_{2} 11$. The next nearest neighbor (NNN) super-exchange constant $\mathrm{J}_{2}^{L D A}=0.04 \mathrm{~K}$, is extremely small, so frustration coupling, if any should be negligible. The ratio of first and second neighbor in-chain coupling is $J_{1}^{L D A} / J_{2}^{L D A} \gtrsim$ 5000 , the largest found in $S=\frac{1}{2}$ chain system. The ratio of in-chain to the strongest (frustrating) inter-chain coupling is $J_{1}^{L D A} / J_{i c 4}^{L D A} \gtrsim 1000$, which is two orders of magnitude larger than in $\mathrm{Sr}_{2} \mathrm{Cu}\left(\mathrm{PO}_{4}\right)_{2}\left(J_{1}^{L D A} / J_{i c}^{L D A} \sim\right.$ $70)^{11}$ and one order of magnitude larger than $\mathrm{Sr}_{2} \mathrm{CuO}_{3}$ $\left(J_{1}^{L D A} / J_{i c}^{L D A} \sim 500\right)^{8,25}$ and making $\mathrm{K}_{2} \mathrm{CuP}_{2} \mathrm{O}_{7}$ an even better realization of $1 \mathrm{D}$ HAF behavior than the $\mathrm{Sr}$ analogue. We have estimated the Néel temperature of $\mathrm{K}_{2} \mathrm{CuP}_{2} \mathrm{O}_{7}\left(T_{N}^{K}\right)$ by adapting a simple mean field approximation ${ }^{8}$ and comparing it to the $\mathrm{Sr}$ analogue $\left(T_{N}^{S r}\right)$. Assuming that the anisotropy are the same in both compounds we can write according to Ref. 8

$$
\frac{T_{N}^{K}}{T_{N}^{S r}} \approx \frac{\sqrt{J_{1}^{K} J_{i c}^{K}}}{\sqrt{J_{1}^{S r} J_{i c}^{S r}}}
$$

where, $T_{N}^{S r} \approx 85 \mathrm{mK}^{10}$. Since the incompleteness in the mapping should be the same for both the compounds, we can directly compare the values of $T_{N}$. We have used $J_{1}^{S r}=187 \mathrm{~K}, J_{i c}^{S r}=0.23 \mathrm{~K}$ (geometrical average of the 2 inter-chain exchanges), $J_{1}^{K}=196 \mathrm{~K}$ and $J_{i c}^{K}=$ 
$\sqrt[4]{J_{i c 1}^{L D A} J_{i c 2}^{L D A} J_{i c 3}^{L D A} J_{i c 4}^{L D A}}=0.037 \mathrm{~K}$. Plugging these values in the above equation we get $T_{N}^{K} \approx 37 \mathrm{mK}$, which is a factor of two smaller than the $\mathrm{Sr}$ analogue. This value sets a upper bound, because the value of $J_{i c}$ is calculated using a mean-field approximation. The absence of anomalies in $K(T), 1 / T_{1}(T)$ and the invariant spectral shape down to low temperatures rules out the possibility of LRO down to $2 \mathrm{~K}$, consistent with the theoretical prediction of an extremely low $T_{N}$. In $\mathrm{K}_{2} \mathrm{CuP}_{2} \mathrm{O}_{7}$, each $\mathrm{CuO}_{4}$ plaquettes in one chain has two identical neighbors in each adjacent chains, with the same exchange interactions. For a strong in-chain AFM exchange, this leads to a complete frustration of the inter-chain interactions. Quantum fluctuations are therefore enhanced here which suppresses $T_{N}$ to lower values.

For a $1 \mathrm{D} S=\frac{1}{2} \mathrm{HAF}$, theoretical calculations predict a weak logarithmic decrease of $\chi(T)$ upon approaching $T=0 \mathrm{~K}$ (see Fig. 4). The lower inset of Fig. 4 shows clearly that the decrease we observe in $K(T)$ of $\mathrm{K}_{2} \mathrm{CuP}_{2} \mathrm{O}_{7}$ is much more pronounced . The susceptibility of a $1 \mathrm{D} S=\frac{1}{2} \mathrm{HAF}$ at $T=0$ is exactly known ${ }^{26}$, $\chi(T=0)=\frac{g^{2} \mu_{B}^{2}}{k_{B}\left(J_{1}\right) \pi^{2}}$. Then, $K(T)$ at zero temperature can be written as $K_{\text {theo }}(T=0)=K_{0}+\left[\frac{A_{h f} g^{2} \mu_{B}}{k_{B}\left(J_{1}\right)}\right] \times \frac{1}{\pi^{2}}$. Using the parameters $\left(K_{0}, A_{h f}, g\right.$, and $\left.J_{1}\right)$ determined from our $K(T)$ analysis, $K_{\text {theo }}(T=0)$ was calculated to be $260 \mathrm{ppm}$. However, our experimental value at 2 $\mathrm{K}$ is much lower, only $180 \mathrm{ppm}$, and $K(T)$ is still decreasing steeply with $T$ at this temperature. Thus, at the quantitative level, the theoretically predicted logarithmic term fails to describe our experimentaly observed decrease. A similar feature has been found experimentally in ${ }^{17} \mathrm{O} \mathrm{NMR}$ on $\mathrm{Sr}_{2} \mathrm{CuO}_{3}$ below $T / J_{1} \simeq 0.015^{30}$ and ${ }^{31} \mathrm{P} \mathrm{NMR}$ on $(\mathrm{Sr}, \mathrm{Ba}){ }_{2} \mathrm{Cu}\left(\mathrm{PO}_{4}\right)_{2}$ below $T / J_{1} \simeq 0.003 . \frac{9}{} \mathrm{In}$ both the cases, it is argued that the decrease is unrelated to the onset magnetic order or a spin-Peierls transition. In $\mathrm{K}_{2} \mathrm{CuP}_{2} \mathrm{O}_{7}$, we neither saw any indication of ordering in $1 / T_{1}(T)$ nor in $C_{p}(T)$. Similarly there is no signature of exponential decrease (or singlet ground state) observed in $1 / T_{1}(T)$ as is expected for a spin-Peierls transition. One possibility to account for this drop in $K(T)$ is the presence of DM interaction arising from the fact that in $\mathrm{K}_{2} \mathrm{CuP}_{2} \mathrm{O}_{7}$ there is no center of inversion symmetryrelating two neighboring copper atoms along the chain. In the presence of DM interaction, application of a magnetic field parallel to the DM vector opens a gap $\Delta$ in the magnetic excitation spectra. Since in a first approximation $\Delta$ increases with $B$ to the power $2 / 3$, it shall not be visible in the $B=0$ specific heat data or the low field susceptibility shown in Fig. 2. However, further experiments are needed to confirm or discard this explanation.

In NMR valuable information on the dynamic of lowenergetic spin excitations can be gained from the analysis of the temperature dependence of nuclear spin-lattice relaxation rates. Therefore, it is essential to analyze $1 / T_{1}(T)$ carefully which yields information about the imaginary part of the dynamic susceptibility $\chi(\mathbf{q}, \omega)$. Thus $1 / T_{1}$ should include contributions from both the uniform $(q=0)$ and staggered $\left(q= \pm \frac{\pi}{a}\right)$ spin fluctuations. A theoretical analysis by Sachdev ${ }^{32}$ shows that the staggered component is dominant at low temperatures $\left(T \ll J_{1}\right)$. Indeed, the uniform component leads to $1 / T_{1} \propto T$, while the staggered component gives $1 / T_{1}$ $=$ constant. Monte Carlo calculations by Sandvik supported the validity of these results over an appropriate temperature range ${ }^{33}$ As shown in Fig. 5, our experimentally observed constant $1 / T_{1}$ at low temperatures $(2 K \leq T \leq 30 K)$ suggests the dominance of staggered fluctuations at low temperatures. The ${ }^{31} \mathrm{P}$ form factor for such systems is defined in Ref. 9. Since ${ }^{31} \mathrm{P}$ is located symmetrically between the $\mathrm{Cu}$ ions, the fluctuations are expected to be filtered out provided the hyperfine couplings are equal. Moreover in this case we still have a significant remnant contribution from $q= \pm \pi / a$ which plays a dominant role at low temperatures. The possible origin of the remnant staggered fluctuations could be the unequal hyperfine couplings. In fact, such features have been previously observed in a few other $1 \mathrm{D} S=\frac{1}{2} \mathrm{HAF}$ systems where $J_{1} \gg T_{N}, 9,13,30,34,35$ The strong increase of $1 / T_{1}$ with $\mathrm{T}$ above $30 \mathrm{~K}$ could be attributed to the uniform fluctuations. When the dominant contribution is from $q=0$, then one expects a constant $1 /\left(K T_{1} T\right)$. In $\mathrm{K}_{2} \mathrm{CuP}_{2} \mathrm{O}_{7}$, for $T \geq 30 \mathrm{~K}$, we rather observe a weak temperature dependency (inset of Fig. 5) which might be due to some remanent contributions from the staggered fluctuations and/or some additional relaxation mechanisms. This weak temperature dependence as well as the reduction of the slope in the plot $1 / T_{1}$ versus $T$ above $180 \mathrm{~K}$ might be related to spin diffusion as observed in $\alpha-\mathrm{VO}\left(\mathrm{PO}_{3}\right)_{2}$ above $J_{1} 38$.

The relaxation rate due to staggered fluctuations can be calculated following the prescription of Barzykin ${ }^{36}$. For the purpose of comparison of theory with experiment he defined the normalized dimensionless NMR spinlattice relaxation rate at low-temperature $\left(1 / T_{1}\right)_{\text {norm }}=$ $\frac{\hbar J_{1}}{A_{t h}^{2} T_{1}} \approx 0.3$, where $A_{t h}$ is $A_{h f}(2 h \gamma / 2 \pi)$. Assuming the fluctuations to be correlated because of the exchange $J_{1}$ along the chains, $1 / T_{1}$ can be written as $1 / T_{1}=\frac{0.3}{\hbar J_{1} / A_{t h}^{2}}$. Using this expression, $\left(1 / T_{1}\right)$ at the ${ }^{31} \mathrm{P}$ site was calculated to be about $129 \mathrm{sec},{ }^{-1}$ whereas our experimental value is $50 \mathrm{sec}^{-1}$ in the $2 \mathrm{~K} \leq T \leq 30 \mathrm{~K}$ range. The experimental value is about two times smaller than the theoretical value, likely due to the effect of the geometrical form factor. Further on a weak logarithmic increase in $1 / T_{1}$ is theoretically expected at low temperatures $\underline{36}$ In the present case, our measurements were not done down to low enough temperatures to rise this contribution above our experimental error.

\section{CONCLUSION}

In conclusion, our experimental and theoretical studies of $\mathrm{K}_{2} \mathrm{CuP}_{2} \mathrm{O}_{7}$ demonstrate that this compound presents a very uniform and strongly $1 \mathrm{D} S=\frac{1}{2} \mathrm{HAF}$ chain system. 
Our NMR, specific heat and susceptibility measurements show good agreement with theoretical predictions for a 1D $S=\frac{1}{2}$ HAF chain. Thus, the temperature dependence of the NMR shift $K$ can be fitted in good agreement with model calculations giving $J_{1} \simeq(141 \pm 5) \mathrm{K}$. A calculation of the exchange interactions using a TBM fitted to the results of $a b$ initio LDA band structure calculations, subsequently mapped onto a Heisenberg model, leads to a quite similar value of $J_{1} \simeq 190 \mathrm{~K}$ and evidences extremely weak NNN interactions as well as extremely weak and "frustrated" inter-chain exchange of the order of $J^{i c} \sim 0.1 \mathrm{~K}$, in contrast to $\mathrm{Sr}_{2} \mathrm{Cu}\left(\mathrm{PO}_{4}\right)_{2}$ with $J^{i c} \sim 9$ $\mathrm{K}^{11}$ and to $\mathrm{Sr}_{2} \mathrm{CuO}_{3}$ "non-frustrated" J $J^{i c} \sim 10 \mathrm{~K}^{8}$. The absence of any evidence for magnetic order above $2 \mathrm{~K}$ in the experimental data confirms the weakness of the interchain exchanges. Using the TBM results, a rough estimate of the Neél temperature results in a value of $T_{N} \sim$ $37 \mathrm{mK}$, only. $\mathrm{K}_{2} \mathrm{CuP}_{2} \mathrm{O}_{7}$ has the smallest in-chain as well as frustrating smallest inter-chain exchanges which do not suppress quantum fluctuations, thereby making this system an even better example of a $1 \mathrm{D} S=\frac{1}{2} \mathrm{HAF}$ system than the compounds reported so far.

We further studied the magnetic fluctuations by analysing the temperature dependence of $1 / T_{1}$. At low temperatures $1 / T_{1}$ remains constant, in reasonable agreement with Sachdev's predictions for 1D $S=\frac{1}{2}$ HAF system where relaxation is dominated by $q= \pm \pi / a$ at low $T$. Below $5 \mathrm{~K}$, we obtained a decrease of the NMR shift $K$ which is more pronounced than that expected for a $1 \mathrm{D} S=\frac{1}{2}$ HAF. It's origin is not yet clear, but might be due to DM interactions.

\section{Acknowledgments}

We thank to A. V. Mahajan, F. Haarmann for their critical suggestions on NMR. We would like to acknowledge Alois Loidl for some preliminary NMR measurements done in his group laboratory. D.K. and H.R. acknowledge financial support from the 'Emmy-Noetherprogram' of the DFG.
* Electronic address: rosner@cpfs.mpg.de

1 F. D. M. Haldane, Phys. Rev. Lett. 50, 1153 (1983).

${ }^{2}$ H. A. Bethe Z. Phys. 71, 205 (1931).

3 E. Lieb, T. D. Schultz, and D. C. Mattis, Ann. Phys. 16, 407 (1961).

4 L. F. Maththeiss, Phys. Rev. B 49, 14050 (1994).

5 R. Neudert, H. Rosner, S. -L. Drechsler, M. Kielwein, M. Sing, Z. Hu, M. Knupfer, M. S. Golden, J. Fink, N. Nuecker, M. Merz, S. Schuppler, N. Motoyama, H. Eisaki, S. Uchida, M. Domke, and G. Kaindl, Phys. Rev. B 60, 13413 (1999).

6 T. Ami, M. K. Crawford, and R. L. Harlow, Z. R. Wang, D. C. Johnston, Q. Huang, and R. W. Erwin, Phys. Rev. B 51, 5994 (1995).

7 N. Motoyama, H. Eisaki, and S. Uchida, Phys. Rev. Lett. 76, 3212 (1996); M. Takigawa, N. Motoyama, H. Eisaki, and S. Uchida, ibid. 76, 4612 (1996).

8 H. Rosner, H. Eschrig, R. Hyan, S. -L. Drechsler, and J. Malek, Phys. Rev. B 56, 3402 (1997).

9 R. Nath, A. V. Mahajan, N. Buttgen, C. Kegler, and A. Loidl, and J. Bobroff, Phys. Rev. B 71, 174436 (2005).

10 Alexei A. Belik, S. Uji, T. Terashima, and E. Takayama, J. Solid State chem. 178, 3461 (2005).

11 M. D. Johannes, J. Richter, S.-L. Drechsler, and H. Rosner, Phys. Rev. B 74, 174435 (2006).

12 S. S. Salunke, M. A. H. Ahsan, R. Nath, A. V. Mahajan, and I. Dasgupta, Phys. Rev. B 76, 085104 (2007).

13 A. V. Mahajan, R. Nath, N. Buttgen, C. Kegler, A. Loidl, and J. Bobroff, Physica B, 378, 1148 (2006).

14 A. ElMaadi, A. Boukhari, and E. M. Holt, J. Alloys and Comps, 223, 13 (1995).

15 R. Nath, A. V. Mahajan, N. Buttgen, C. Kegler, J. Hemberger, and A. Loidl, J. Phys. Cond. Matt. 18, 4285 (2006).

16 L. Alexander et al., (private communication)

17 K. Koepernik, and H. Eschrig, Phys. Rev. B textbf59, 1743 (1999).

18 I. Opahle, K. Koepernik, and H. Eschrig, Phys. Rev. B 60,
14035 (1999)

19 H. Eschrig, Optimized LCAO Method (Springer-Verlag, Berlin, 1989).

20 J.P. Perdew and Y. Wang, Phys. Rev. B 45, 13244 (1992).

21 M. Oshikawa and I. Affleck, Phys. Rev. Lett. 79, 2883 (1997).

22 I. Affleck and M. Oshikawa, Phys. Rev. B 60, 1038 (1999).

23 D. C. Dender, D. Davidovic, Daniel H. Reich, Collin Broholm, Kim Lefmann, and G. Aeppli, Phys. Rev. B 53, 2583 (1996)

24 R. Feyerherm, A. Abens, D. Günther, T. Ishida, M. Meissner, M. Meschke, T. Nogami, and M. Steiner, J. Phys. Cond. Matt. 12, 8495 (2000).

25 Taking the measure of $J_{1}=190 \mathrm{meV}$ from the magnetic susceptibility experiment of Ref. 7 and the empirical $J_{\perp}=0.2$ $\mathrm{meV}$, calculated from the ordering temperature $T_{N}=5 \mathrm{~K}$, using coupled quantum spin chain approach as in Ref. 8, we find $J_{1} / J_{\perp} \sim 600$, still one order of magnitude smaller than $\mathrm{K}_{2} \mathrm{CuP}_{2} \mathrm{O}_{7}$.

26 D. C. Johnston, R. K. Kremer, M. Troyer, X. Wang, A. Klümper, S. L. Bud'ko, A. F. Panchula, and P. C. Canfield, Phys. Rev. B 61, 9558 (2000).

27 Making $g$ as a free parameter gives a reduced value of about 1.8. Nevertheless in either case it does not lead to a noticeable change in the value of $J_{1}(\sim 130 \mathrm{~K})$.

28 B. Schmidt, V. Yushankhai, L. Siurakshina, and P. Thalmeier, Eur. Phys. J. B 3243 (2003).

29 A. Klumper, The European Physical Journal B, 5, 677 (1998).

${ }^{30}$ K. R. Thurber, A. W. Hunt, T. Imai, and F. C. Chou, Phys. Rev. Lett. 87, 247202 (2001).

31 S. Eggert and I. Affleck Phys. Rev. Lett. 75, 934 (1995).

32 S. Sachdev, Phys. Rev. B 50, 13006 (1994).

33 A. W. Sandvik, Phys. Rev. B 52, 9831 (1995).

34 M. Takigawa, N. Motoyama, H. Eisaki, and S. Uchida, Phys. Rev. Lett. 76, 4612 (1996).

35 M. Takigawa, O. A. Starykh, A. W. Sandvik, and R. R. P. 
Singh, Phys. Rev. B 56, 13681 (1997).

36 V. Barzykin, Phys. Rev. B 63, 140412 (2001).

37 V. Yu. Irkhin and A. A. Katanin, Phys. Rev. B 61, 6757 (1999).
38 J. Kikuchi, N. Kurata, K. Motoya, T. Yamauchi, and Y. Ueda, J. Phys. Soc. Jpn. 70, 2765 (2001). 


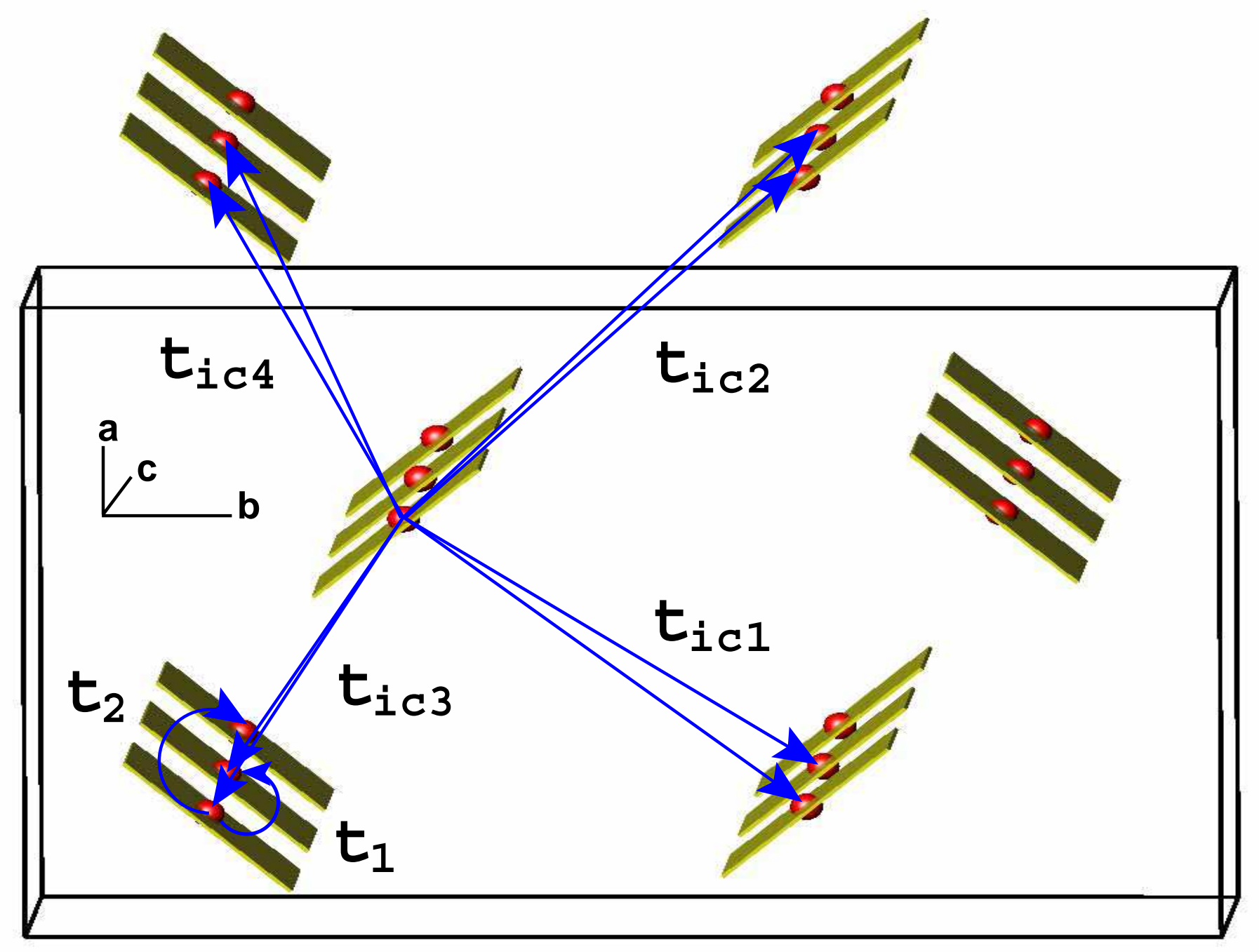

